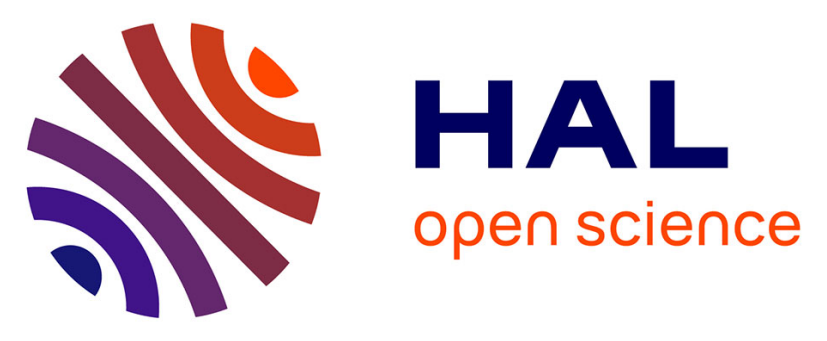

\title{
Colonic neuropathology is not associated with autonomic dysfunction in Parkinson's disease
}

\author{
Laurène Leclair-Visonneau, Thomas Clairembault, Christelle Volteau, \\ Guillaume Chapelet, Séverine Le Dily, Fabienne Vavasseur, Emmanuel Coron, \\ Cécile Préterre, Michel Neunlist, Yann Péréon, et al.
}

\section{To cite this version:}

Laurène Leclair-Visonneau, Thomas Clairembault, Christelle Volteau, Guillaume Chapelet, Séverine Le Dily, et al.. Colonic neuropathology is not associated with autonomic dysfunction in Parkinson's disease. Parkinsonism \& Related Disorders, 2019, 61, pp.224 - 227. 10.1016/j.parkreldis.2018.09.021 . hal-03485668

\section{HAL Id: hal-03485668 \\ https://hal.science/hal-03485668}

Submitted on 20 Dec 2021

HAL is a multi-disciplinary open access archive for the deposit and dissemination of scientific research documents, whether they are published or not. The documents may come from teaching and research institutions in France or abroad, or from public or private research centers.
L'archive ouverte pluridisciplinaire HAL, est destinée au dépôt et à la diffusion de documents scientifiques de niveau recherche, publiés ou non, émanant des établissements d'enseignement et de recherche français ou étrangers, des laboratoires publics ou privés.

\section{(ㅇ)(1) $\$$}

Distributed under a Creative Commons Attribution - NonCommerciall 4.0 International 


\section{Colonic neuropathology is not associated with autonomic dysfunction in}

\section{Parkinson's disease}

Laurène Leclair-Visonneau, MD, PhD*1,2,3,4, Thomas Clairembault, $\mathrm{PhD}^{1,2}$, Christelle Volteau, $\mathrm{MS}^{5}$, Guillaume Chapelet, MD²,6, Séverine Le Dily, $\mathrm{MS}^{3}$, Fabienne Vavasseur ${ }^{3}$, Emmanuel Coron ${ }^{1,2,3,7}$, Cécile Préterre, $\mathrm{MD}^{1,2,3,8}$, Michel Neunlist, $\mathrm{PhD}^{1,2,7}$, Yann Péréon, $\mathrm{MD}, \mathrm{PhD}^{2,4}$, and Pascal Derkinderen, $\mathrm{MD}, \mathrm{PhD}^{1,2,3,8}$

${ }^{1}$ Inserm, U1235, Faculté de Médecine, 1 rue Gaston Veil, F-44035 Nantes, France

²University Nantes, Faculté de Médecine, 1 rue Gaston Veil, F-44035 Nantes, France

${ }^{3}$ Inserm, CIC-04, CHU de Nantes, Bd Jacques Monod, F-44093 Nantes, France

${ }^{4} \mathrm{CHU}$ Nantes, Department of Clinical Neurophysiology, Bd Jacques Monod, F-44093 Nantes, France

${ }^{5} \mathrm{CHU}$ Nantes, Plateforme de Biométrie, département Promotion DRCl, 1 Place Alexis Ricordeau, F-44093 Nantes, France

${ }^{6} \mathrm{CHU}$ Nantes, Clinical Gerontology Department, Bd Jacques Monod, F-44093 Nantes, France ${ }^{7} \mathrm{CHU}$ Nantes, Institut des Maladies de I'Appareil Digestif, 1 Place Alexis Ricordeau, F-44093 Nantes, France

${ }^{8} \mathrm{CHU}$ Nantes, Department of Neurology, Nantes, Bd Jacques Monod, F-44093 Nantes, France

\section{*Corresponding author:}

Laurène Leclair-Visonneau. Department of Clinical Neurophysiology, CHU de Nantes, Bd Jacques Monod 44093 Nantes CEDEX 01, France. Tel: 0033240165889; laurene.leclair@chunantes.fr. ORCID ID: 0000-0001-6379-0765 
Keywords: Parkinson's disease; enteric nervous system; colonic biopsy; alpha-synuclein; dysautonomia 


\section{Abstract \\ $\underline{\text { Introduction }}$}

Dysautonomia in Parkinson's disease (PD) has been shown to be associated with disease severity and especially with the occurrence of dementia. One proposed explanation for this finding is that phosphorylated alpha-synuclein histopathology (PASH), the characteristic pathological feature of PD is more diffuse in dysautonomia-associated PD than in disease without dysautonomia, not only in the central nervous system but also in peripheral autonomic networks. The aim of this study was therefore to determine if colonic alphasynuclein histopathology is associated with dysautonomia in PD.

\section{Methods}

A total of 43 PD patients participated in this study. For each patient, two biopsies were taken in the sigmoid colon and analyzed by immunohistochemistry with antibodies against phosphorylated alpha-synuclein and PGP 9.5. All patients had a complete neuropsychological and neurological assessment along with a comprehensive evaluation of dysautonomia with questionnaires (SCOPA-Aut, NMS-Quest, Rome III constipation criteria and dry eye symptoms) and functional tests (pupillometry, Saxon and Schirmer's tests, heart rate variability, orthostatic blood pressure measure and sympathetic skin response).

\section{$\underline{\text { Results }}$}

Colonic PASH was observed in 20/43 PD patients. No differences were observed in autonomic symptoms and testing between patients with and without PASH.

\section{Conclusions}

Although frequent in PD, autonomic dysfunction is not related to colonic PASH. In addition to the existing literature, our findings further suggest that each dysautonomic symptom in 
PD might not be associated with a more severe or diffuse PASH not only in the central nervous system but also in the peripheral autonomic nervous systems.

\section{Introduction}

The pathological hallmark of Parkinson's disease (PD) is the intraneuronal accumulation of phosphorylated alpha-synuclein in the brain as the primary component of Lewy bodies and neurites. A substantial body of evidence demonstrating peripheral autonomic nervous system pathology has challenged the traditional view of PD as a disorder of the central nervous system [1]. Remarkably, some of the end organs innervated by these peripheral autonomic nervous networks are accessible to biopsies, making them a putative original source of histopathological markers for PD [2]. Among these organs, much attention has been focused on the diagnostic and prognostic potential of colonic biopsies, not only because gastrointestinal symptoms are frequent non-motor symptoms of PD but also because colonic biopsy cancer screening is performed on a large subset of the normal middle-aged population [2].

As discussed in our previous report [3], there has been considerable debate regarding the immunohistochemical method that should be used for the detection of aggregated and phosphorylated alpha-synuclein in colonic biopsies. Most existing studies on gastrointestinal biopsies in PD have been performed on formalin-fixed, paraffin-embedded (FFPE) tissue, which has the main advantages of being readily accessible to most hospital-based laboratories and to allow retrospective analyses. Nevertheless, these studies have had conflicting results regarding the sensitivity and specificity of FFPE colonic biopsies for the detection of pathological alpha-synuclein [2]. This overall lack of specificity of FFPE biopsies prompted us to use another approach for the detection of phosphorylated alpha-synuclein 
by microdissecting the biopsy in order to collect whole-mounts of submucosa [4]. Using this approach, we previously showed that phosphorylated alpha-synuclein histopathology (PASH) was detected in the sigmoid colon in approximately half of PD patients when 2 biopsies per subject were analyzed [5]. In a more recent survey, we observed a significant higher proportion of PASH in PD patient with rapid eye movement (REM) sleep behavior disorder (RBD), a sleep disorder that has been consistently associated with poor PD prognosis [3]. The aim of the current study was to extend these results by determining if autonomic dysfunction, another factor associated with disease severity, was also linked to enteric neuropathology in PD.

\section{Patients and methods}

Study population

The 43 PD patients evaluated in the current research were initially recruited to study the association between RBD and enteric PASH [3] and to perform a comprehensive evaluation of autonomic dysfunction in PD [6]. This study was carried out in accordance with the Declaration of Helsinki, conducted with the approval of the local Ethical Committee (Comité de protection des personnes Ouest VI, France) and registered on ClinicalTrials.gov (identifier NCT01748409). Each participant gave written informed consent.

\section{Endoscopic procedure and tissue collection}

For each subject, 2 biopsies were taken using standard biopsy forceps without needle (FB220U, Olympus co., Japan) during the course of a rectosigmoidoscopy. The 2 biopsies intended for immunohistochemistry were immediately immersed in $4^{\circ} \mathrm{C}$ saline or Hank's 
Buffered Salt Solution (HBSS, Sigma, Saint Quentin Fallavier, France) and kept on ice for no more than an hour until dissection.

\section{Dissection of sigmoid biopsies, whole-mount preparations}

Biopsies were transferred in a Sylgard-coated Petri dish filled with HBSS at $4^{\circ} \mathrm{C}$, then stretched and pinned flat under a stereomicroscope. The submucosa was mechanically separated from the mucosa with watchmaker's forceps and fixed in phosphate buffered saline (PBS) with 4\% (v/v) paraformaldehyde (Sigma) for 3h at room temperature or overnight at $4^{\circ} \mathrm{C}$. After fixation, the samples were rinsed 3 times for 10 min with PBS and kept at $4^{\circ} \mathrm{C}$ in PBS with $1 \%(\mathrm{w} / \mathrm{v})$ sodium azide $\left(\mathrm{PBS} / \mathrm{NaN}_{3}\right)$ until further use.

\section{Immunohistochemistry}

Each whole-mount preparation of submucosa obtained from a single biopsy was

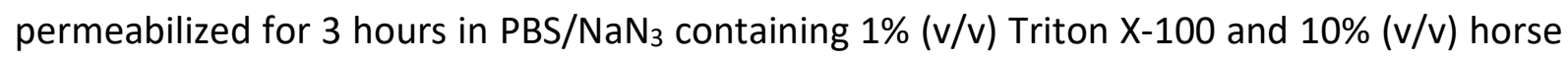
serum and then incubated with antibodies against phosphorylated alpha-synuclein (1:5000, WAKO, Osaka, Japan) and PGP9.5 (1:10,000; Ultraclone Limited, UK). Suitable secondary antibodies conjugated to Alexa Fluor 488 and 594 were used (Invitrogen, Cergy-Pontoise, France). Specimens were viewed under a Zeiss Axiovert 200M microscope fluorescence microscope. Each fragment of submucosa was entirely scanned using the MosaiX module of Axovision software (Zeiss, Göttingen, Germany). The generated image was used as a map to analyze the whole biopsy for the presence of PASH (supplementary figure $1 \mathrm{~A}$ ).

A biopsy was considered positive when containing at least one structure immunoreactive for both phosphorylated alpha-synuclein and PGP9.5 (Supplementary Figure 1B). A patient was noted as positive $(\mathrm{PASH}+)$ when at least one of the two biopsies contained PASH. Based on our previous study performed in 29 PD patients who had 4 
colonic biopsies ( 2 in the ascending and 2 in the descending/sigmoid colon) [4], patients classified into PASH+ and PASH- after analysis of 2 descending colon biopsies could be considered as having high (severe) colonic alpha-synuclein and low (absent or moderate) colonic alpha-synuclein burden, respectively. In this study, we indeed showed that $8 / 29$ patients who had no PASH in the descending colon were also negative in the ascending colon (and therefore classified as 'without alpha-synuclein pathology'), while 10/29 PASH+ patients in the descending colon were also positive in the ascending colon ( $\geq 2 / 4 \mathrm{PASH}+$ biopsies and therefore classified as with 'severe alpha-synuclein pathology') [4]. All 11 remaining patients had $1 / 4 \mathrm{PASH}+$ biopsies either in the ascending or descending colon and were therefore classified as having 'moderate alpha-synuclein pathology'.

\section{Motor and cognitive function evaluation}

All 43 patients were evaluated using the Unified Parkinson's disease Rating Scale part III (UPDRS-III) in ON state. Neuropsychological evaluation was carried out using the minimental state examination (MMSE), Montreal cognitive assessment (MoCA) and Mattis dementia rating scale (MDRS).

\section{Autonomic nervous system evaluation}

Autonomic symptoms were collected with SCOPA-Aut (Scales for Outcomes in PD-autonomic symptoms), NMS-Quest (Non-motor symptoms questionnaire), Rome III constipation criteria and an additional question on dry eye. Autonomic functional testing was carried out as previously described [6]. Briefly, parasympathetic division function was evaluated with pupil constriction parameters (constriction amplitude, latency and velocity), tear secretion (Schirmer's test), saliva production (Saxon test) and heart rate variability. Sympathetic 
division assessment comprised pupil dilation parameters (maximal amplitude and re-dilation velocity), orthostatic hypotension and sudomotor function (sympathetic skin response $(\mathrm{SSR}))$.

\section{$\underline{\text { Statistics }}$}

Continuous variables were expressed as the mean \pm standard deviation and categorical data as numbers and percentage. Continuous variables were compared between groups (patients with and without PASH) using the two-sample Student test or the non-parametric Wilcoxon test (when normality assumption could not be assumed); and the Chi-2 test or Fisher test were used for categorical variables (SAS software ${ }^{\circledR}$ version 9.4). For all statistical tests $\mathrm{p}<0.05$ was deemed significant.

\section{Results}

As previously reported [5], among the 43 PD patients who were analyzed, 20 (47\%) were PASH+. PASH formed an irregular line that was sometimes interrupted but clearly followed the course of the axon and thus morphologically reminiscent of Lewy neurites (Supplementary Figure 1B).

Clinical features, including age, gender, disease duration, levodopa treatment, motor severity (UPDRS-III in ON state) and cognitive impairment (MMSE, MoCA and MDRS), did not differ between PD patients with or without colonic PASH (Table 1). Autonomic symptoms (SCOPA-Aut, NMS-Quest, dry eye symptoms) were similar between PASH+ and PASHpatients (Table 2). Of note, was the absence of association between constipation according to Rome III criteria and colonic PASH (Table 2). A recent report showed that questions 10 (hard stools) and 11 (straining) of Rome III functional constipation questionnaires correlated 
with the objective evaluation of total transit time using radio opaque marker technique [7], but we nevertheless did not observe any association between hard stools or straining for defecation and colonic PASH ( $p=0.78$ and 0.42 , respectively).

Functional testing of parasympathetic and sympathetic autonomic nervous systems revealed no difference between the two groups (Table 2).

\section{Discussion}

In accordance with our previous findings, we found PASH in $47 \%$ of PD patients when two descending/sigmoid colon biopsies were analyzed [5]. When patients were classified into 2 groups, with or without PASH, we did not observe any association between the presence of colonic neuropathology and autonomic dysfunction, motor or cognitive impairment.

Although there have been numerous reports on the detection of pathological alphasynuclein in gastrointestinal biopsies in PD, relatively little attention has been paid to clinical-pathological correlations. Most of the existing studies, which focused on disease duration, motor symptoms (evaluated by UPDRS III and Hoehn and Yahr scales) and constipation, did not find any correlation between these parameters and the enteric pathological burden $[4,8-10]$. In a recent report carried out in 38 PD subjects who had gastrointestinal biopsies taken during the course of gastroduodenoscopy and colonoscopy, Chung et al. did not observe any relationship between enteric synucleinopathy and nonmotor features using the non-motor symptom assessment scale [8]. Despite a more comprehensive and accurate evaluation of autonomic functions by physiological means (except for gastrointestinal dysfunction which was evaluated by questionnaires), we were also unable to find any link between the presence of enteric phospho-alpha synuclein deposits and autonomic dysfunction, either at the parasympathetic or sympathetic level. A 
recent study, which analyzed the clinical data from 100 consecutive patients with an autopsy-confirmed diagnosis of PD, showed that earlier development of orthostatic hypotension, urinary symptoms, upper gastrointestinal tract symptoms, constipation and sweating abnormalities were independent determinants of a more rapid disease progression along with shorter survival [11]. One possible explanation proposed for this finding is that alpha-synuclein pathology is more extensive in dysautonomia-associated PD than in disease without autonomic dysfunction, not only in the CNS but also in peripheral autonomic networks. Nevertheless, after performing a semi-quantitative evaluation of Lewy pathology in the brainstem, limbic system and cortex of these 100 PD cases no correlations between the pathological burden and dysautonomic symptoms were observed [11]. Despite being limited by a patholological analysis restricted to the sigmoid/descending colon, our findings by showing no association between colonic PASH and autonomic dysfunction, provide additional clue that PD patients with dysautonomia might not have a more diffuse alphasynuclein neuropathology. By contrast to autonomic dysfunction, we found an association between colonic PASH and RBD [3], a parasomnia that has been consistently associated with a more severe PD phenotype. In addition to more enteric PASH, PD patients with RBD also have a greater density in PASH in the CNS, not only in cortical but also in brainstem nuclei such as dorsal motor nucleus of the vagus and locus coeruleus [12]. Taken together, these observations suggest that PD patients with RBD but not those with autonomic dysfunction have more widespread neuropathological changes, not only in the brain but also in the peripheral autonomic nervous system.

Since the identification of alpha-synuclein as a major component of Lewy bodies and neurites, there has been considerable debate about the pathophysiological significance of alpha-synuclein deposits in PD. Several authors have suggested that Lewy pathology detects 
neurons that are dysfunctional and therefore that the mere presence of alpha-synuclein deposits in a tissue would be sufficient to induce organ specific dysfunction, while others argue that neurons with alpha-synuclein deposits may not be in a state of PD-related dysfunction. The absence of relationship between PASH and constipation observed in our study, using either the Rome III questionnaire or two specific questions (straining for defecation or hard stools), would lend support to the latter assumption. Moreover, aside from neuronal dysfunction, intestinal microbiota may explain non-motor symptoms, in particular constipation in PD.

In conclusion, our results provide evidence that colonic PASH is not associated with autonomic symptoms and dysfunction. Together with previous findings [9], they also suggest that dysautonomia in PD is complex and not merely related to a more diffuse alphasynuclein pathology.

\section{Acknowledgements}

Nantes University Hospital was the study promoter. This work was supported by a grant from Nantes University Hospital (Appel d'offre interne 2012, Grant number RC12_0264) and France Parkinson.

\section{Conflict of interest}

The authors declare that they have no conflict of interest.

\section{Appendix}

Supplementary Figure 1. (A) Whole mount of submucosa is stained with PGP9.5 antibody. PGP9.5 staining revealed the architecture of the submucosal plexus characterized by ganglia 
connected together via interganglionic fiber strands. (B) Phosphorylated alpha-synuclein

histopathology (PASH) in the submucosa of a PD patient. A biopsy taken in the sigmoid colon of a PD patient was microdissected and analyzed by immunohistochemistry. The representative photomicrograph shows multiple PASH in a whole-mount of submucosa immunoreactive for both phosphorylated alpha-synuclein and PGP 9.5.

\section{References}

[1] K. Wakabayashi, F. Mori, K. Tanji, S. Orimo, H. Takahashi, Involvement of the peripheral nervous system in synucleinopathies, tauopathies and other neurodegenerative proteinopathies of the brain, Acta Neuropathol. 120 (2010) 1-12. doi:10.1007/s00401010-0706-x.

[2] J.M. Lee, P. Derkinderen, J.H. Kordower, R. Freeman, D.G. Munoz, T. Kremer, W. Zago, S.J. Hutten, C.H. Adler, G.E. Serrano, T.G. Beach, The Search for a Peripheral Biopsy Indicator of $\alpha$-Synuclein Pathology for Parkinson Disease, J. Neuropathol. Exp. Neurol. 76 (2017) 2-15. doi:10.1093/jnen/nlw103.

[3] L. Leclair-Visonneau, T. Clairembault, E. Coron, S. Le Dily, F. Vavasseur, M. Dalichampt, Y. Péréon, M. Neunlist, P. Derkinderen, REM sleep behavior disorder is related to enteric neuropathology in Parkinson disease, Neurology. 89 (2017) 1612-1618. doi:10.1212/WNL.0000000000004496.

[4] T. Lebouvier, M. Neunlist, S. Bruley des Varannes, E. Coron, A. Drouard, J.-M. N'Guyen, T. Chaumette, M. Tasselli, S. Paillusson, M. Flamand, J.-P. Galmiche, P. Damier, P. Derkinderen, Colonic biopsies to assess the neuropathology of Parkinson's disease and its relationship with symptoms, PLoS ONE. 5 (2010) e12728. doi:10.1371/journal.pone.0012728.

[5] H. Pouclet, T. Lebouvier, E. Coron, S.B. des Varannes, T. Rouaud, M. Roy, M. Neunlist, P. Derkinderen, A comparison between rectal and colonic biopsies to detect Lewy pathology in Parkinson's disease, Neurobiol. Dis. 45 (2012) 305-309. doi:10.1016/j.nbd.2011.08.014.

[6] L. Leclair-Visonneau, L. Magy, C. Volteau, T. Clairembault, S. Le Dily, C. Préterre, A. Peyre, P. Damier, M. Neunlist, Y. Péréon, P. Derkinderen, Heterogeneous pattern of autonomic dysfunction in Parkinson's disease, J. Neurol. 265 (2018) 933-941. doi:10.1007/s00415-018-8789-8.

[7] K. Knudsen, T.D. Fedorova, A.C. Bekker, P. Iversen, K. Østergaard, K. Krogh, P. Borghammer, Objective Colonic Dysfunction is Far more Prevalent than Subjective Constipation in Parkinson's Disease: A Colon Transit and Volume Study, J Parkinsons Dis. 7 (2017) 359-367. doi:10.3233/JPD-161050.

[8] S.J. Chung, J. Kim, H.J. Lee, H.-S. Ryu, K. Kim, J.H. Lee, K.W. Jung, M.J. Kim, M.-J. Kim, Y.J. Kim, S.-C. Yun, J.-Y. Lee, S.-M. Hong, S.-J. Myung, Alpha-synuclein in gastric and colonic mucosa in Parkinson's disease: Limited role as a biomarker, Mov. Disord. 31 (2016) 241-249. doi:10.1002/mds.26473. 
[9] Á. Sánchez-Ferro, A. Rábano, M.J. Catalán, F.C. Rodríguez-Valcárcel, S. Fernández Díez, J. Herreros-Rodríguez, E. García-Cobos, M.M. Álvarez-Santullano, L. López-Manzanares, A.J. Mosqueira, L. Vela Desojo, J.J. López-Lozano, E. López-Valdés, R. Sánchez-Sánchez, J.A. Molina-Arjona, In vivo gastric detection of $\alpha$-synuclein inclusions in Parkinson's disease, Mov. Disord. 30 (2015) 517-524. doi:10.1002/mds.25988.

[10] M. Barrenschee, D. Zorenkov, M. Böttner, C. Lange, F. Cossais, A.B. Scharf, G. Deuschl, S.A. Schneider, M. Ellrichmann, A. Fritscher-Ravens, T. Wedel, Distinct pattern of enteric phospho-alpha-synuclein aggregates and gene expression profiles in patients with Parkinson's disease, Acta Neuropathol Commun. 5 (2017) 1. doi:10.1186/s40478016-0408-2.

[11] E. De Pablo-Fernandez, C. Tur, T. Revesz, A.J. Lees, J.L. Holton, T.T. Warner, Association of Autonomic Dysfunction With Disease Progression and Survival in Parkinson Disease, JAMA Neurol. 74 (2017) 970-976. doi:10.1001/jamaneurol.2017.1125.

[12] R.B. Postuma, C.H. Adler, B.N. Dugger, J.G. Hentz, H.A. Shill, E. Driver-Dunckley, M.N. Sabbagh, S.A. Jacobson, C.M. Belden, L.I. Sue, G. Serrano, T.G. Beach, REM sleep behavior disorder and neuropathology in Parkinson's disease, Mov. Disord. 30 (2015) 1413-1417. doi:10.1002/mds.26347. 
$\underline{\text { Table 1. Main clinical characteristics of PD patients with (PASH }+ \text { ) and without (PASH-) colonic }}$ $\underline{\text { PASH. }}$

\begin{tabular}{|l|l|l|l|}
\hline & PASH+ & PASH- & p-value \\
\hline no., \% & $20(47 \%)$ & $23(53 \%)$ & \\
\hline Gender, \% male & $16(80 \%)$ & $12(52 \%)$ & 0.11 \\
\hline Age, y & $61.6(6.9)$ & $59.8(8.5)$ & 0.47 \\
\hline Disease duration, y & $9.8(6.9)$ & $8.0(6.3)$ & 0.37 \\
\hline L-Dopa lifetime cumulative dose, kg & $1.4(1.1)$ & $0.9(1.3)$ & 0.16 \\
\hline L-Dopa equivalent daily dose, g & $1.0(0.5)$ & $0.7(0.6)$ & 0.12 \\
\hline UPDRS-III 'on' & $24.3(13.2)$ & $19.7(10.9)$ & 0.23 \\
\hline Neuropsychological tests & & & \\
\hline MMSE (0-30) & $27.6(2.2)$ & $28.2(1.8)$ & 0.29 \\
\hline MoCA (0-30) & $24.8(4.2)$ & $26.0(3.2)$ & 0.27 \\
\hline MDRS (0-144) & $137.0(5.2)$ & $139.2(4.9)$ & 0.15 \\
\hline
\end{tabular}

Abbreviations: MDRS: Mattis dementia rating scale; MMSE: Mini-Mental State Examination;

MoCA = Montreal Cognitive Assessment; PASH = phosphorylated alpha-synuclein

histopathology; UPDRS-III = Unified Parkinson's Disease Rating Scale part III.

Continuous variables are mean (standard deviation) and categorical data are numbers (percentage). 
$\underline{\text { Table 2. Autonomic symptoms and altered functional testing in PD patients with (PASH+) and }}$ without (PASH-) colonic PASH.

\begin{tabular}{|c|c|c|c|}
\hline & PASH+ & PASH- & $p$-value \\
\hline no., $\%$ & $20(47 \%)$ & $23(53 \%)$ & \\
\hline SCOPA-Aut (0-69) & $22.4(6.4)$ & $22.0(10.7)$ & 0.89 \\
\hline NMS-Quest (0-30) & $13.9(4.8)$ & $12.4(4.6)$ & 0.29 \\
\hline Constipation Rome III criteria, n (\%) & $12(60.0 \%)$ & $13(56.5 \%)$ & 0.82 \\
\hline Dry eye symptoms & $8(40 \%)$ & $11(47.8 \%)$ & 0.17 \\
\hline Parasympathetic testing & & & \\
\hline Pupillometry (constriction) & $3(15.8 \%)$ & $6(26.1 \%)$ & 0.48 \\
\hline Schirmer's test & $13(65 \%)$ & $17(74 \%)$ & 0.60 \\
\hline Saxon test (saliva) & $9(45.0 \%)$ & $14(60.9 \%)$ & 0.30 \\
\hline Heart rate variability & $5(26.3 \%)$ & $7(31.8 \%)$ & 0.70 \\
\hline Sympathetic testing & & & \\
\hline Pupillometry (dilation) & $0(0)$ & $3(13.0 \%)$ & 0.24 \\
\hline Orthostatic hypotension & $14(70.0 \%)$ & $19(82.6 \%)$ & 0.47 \\
\hline Sympathetic skin response & $1(5.3 \%)$ & $3(13.0 \%)$ & 0.74 \\
\hline
\end{tabular}

Abbreviations: NMS-Quest: Non-motor symptoms questionnaire; PASH: phosphorylated alpha-synuclein histopathology; SCOPA-Aut =Scales for Outcomes in PD-autonomic 
symptoms. Continuous variables are mean (standard deviation) and categorical data are numbers (percentage). 